\title{
EFFECTS OF PRE-DETERMINED LEVEL OF FOLIC ACID SUPPLEMENT ON PERFORMANCE AND CARCASS CHARACTERISTICS OF BROILER CHICKENS
}

\author{
Victoria Nnenna MEREMIKWU ${ }^{\star \Delta}$ and Esther Darlington IZUKI ${ }^{2}$ \\ ${ }^{1}$ Department of Animal Science, Faculty of Agriculture and Forestry, Obubra Campus, Cross River University of Technology, PMB 112, Cross River \\ State, Nigeria \\ 2Department of Animal Science, Faculty of Agriculture, University of Calabar, Cross River State, Nigeria \\ Email: victoriameremikwu@yahoo.com; (D) ORCiD: 0000-0002-3904-8234 \\ supporting Information
}

ABSTRACT: A pre-determined level of folic acid supplement (30 mg per litre of drinking water) was fed for varying durations $(7,10$ and 14 days) from day-one of age to determine the effect on performance and carcass characteristics of broilers. The objective was to confirm the high levels of abdominal fat pads in previous trials with graded levels of folic acid, to clarify the mechanism underlying adipose tissue growth in broilers. Parameters measured were body weight, weight gain, feed intake, feed conversion ratio, folic acid intake, mortality and dressed weight, dressing percentage, carcass cuts and internal organs. Data obtained were analyzed using statistical package for social sciences. The outstanding result of this research was on the conformation of the dressed carcasses of the folic acid treated birds, characterized by expanded abdominal regions filled with large mass of abdominal fat pads. There was no difference between the control and the folic acid birds in other parameters measured, except the group on the longest duration of folic acid supplementation, which had higher feed intake. Folic acid intake increased significantly with increase in the duration of administration. It was concluded that, the large mass of abdominal fat pads of the folic acid birds were as a result of cell multiplication (hyperplasia) due to the fact that folate-mediated one-carbon units transfer reactions support rapid proliferation of cells and are important during periods of active cell division.

Keywords: Abdominal fat, Broiler, Folic acid, Pre-determined level, Supplement.

\section{INTRODUCTION}

Folic acid (Pteroyl-L-glutamic acid) is the synthetic form of a water soluble vitamin (vitamin B9) which occurs naturally as folate. Folate is richly available in dark green vegetables while folic acid is often recommended as a nutritional supplement and for fortification of foods (Chan et al., 2015). Folic acid is referred to as a growth promoting factor because of its role in the production and maintenance of new cells (Wagner 2001; Armando, 2018). It is reported to be particularly important during periods of active cell division such as pregnancy and infancy (Armando, 2018; Tjong and Mohiuddin, 2019). It is essential in cell metabolism because of its use in the biosynthesis of components of nucleic acid needed for cell division (Barry, 2001; Tjong and Mohiuddin, 2019).

Folic acid itself is not biologically active but it is converted to its biological active form tetrahydrofolic acid (THF) after its conversion to dihydrofolic acid by dihydrofolic reductase in the liver (Fernández-Villa et al., 2019). Tetrahydrofolic acid is a cofactor in one-carbon metabolism which is a universal metabolic process that serves to activate and transfer onecarbon units for biosynthetic processes in the body (Barry, 2001). Tetrahydrofolic acid can also be converted to other onecarbon transport forms by serving as a carrier molecule for one -carbon groups e. g. methyl - THF, methylene - THF, methenyl-THF (Fernández-Villa et al., 2019). These folic acid derivatives especially tetrahydrofolic acid serve as coenzymes in one-carbon units transfer reactions during cellular activities. Folate-mediated single-carbon transfer reactions are important in biosynthetic pathways leading to DNA and RNA synthesis, amino acid metabolism, methylation and remethylation reactions (Barry, 2001).

Tetrahydrofolic acid plays key role in DNA synthesis by serving as a direct donor of one-carbon units in the synthesis of components of nucleic acid (the critical base pairs) needed by DNA for replication i.e. the synthesis of DNA from its precursors (thymidine and purine) is dependent on folate-mediated one-carbon unit transfer reactions (Field, 2018). According to Field (2018), folate-mediated one-carbon units transfer reactions support high proliferation rate of normal cells and cancer cells because of many activated precursors of nucleic acid (thymidine and purine). This body of knowledge had led to addition of folic acid to virtually all cell culture media because, it enhances the growth of cells in serum-free culture (Media Experts, 2020). Deficiency of folic acid leads to impaired cell division due to impairment in thymidine and purine synthesis, resulting from impairment in one-carbon metabolism (Greenberg et al., 2011; Field, 2018). In avian species, folic acid deficiency is characterized by poor growth, very poor feathering and anaemic appearance in chicks (Poultry DVM, 2020). Supplemental folic acid nutrition has not been a major concern in the poultry industry. However, some researchers had investigated the growth promoting potentials of folic acid on the growth performance of the broiler chicken. Meremikwu et al. (2008) fed graded levels of folic acid per litre of drinking water for the first five days of age of the broiler. The result of the trial showed enhanced performance in the folic acid treated birds 
over the control, with higher levels of abdominal fat in the folic acid birds than the control. There was however no difference between the folic acid treated birds. A second trial was conducted with the same levels of folic acid supplement as in the previous trial but for a longer period of seven days from day-one of age of the broiler (Meremikwu et al., 2015). The result of the later trial showed a clearer picture of the effect of the different levels of folic acid supplementation on broiler performance. There were conspicuous and significant higher levels of abdominal fat pads in the folic acid treated birds than the control, with $30 \mathrm{mg}$ level of supplementation eliciting a better effect than the other two levels (15 and $45 \mathrm{mg}$ ) in all the performance parameters measured.

The high levels of abdominal fat pads in the folic acid treated birds recorded in the two previous trials calls for a further research to clarify the mechanism underlying adipose tissue development in broilers. This may enhance efforts to develop measures to reduce the accumulation of excess body fat in broilers. This is necessary since excessive accumulation of fat in adipose tissue of broilers have been a major problem in the broiler industry. This is because high body fat in broilers is associated with obesity and several metabolic disorders especially those affecting the cardiovascular system which are responsible for a majority of flock mortality in broilers (Whitehead, 2001). The use of abdominal fat content has been reported to be very useful in reducing fat deposition in broilers in the short- term than the use of selection procedures in the long-term. This is because, abdominal fat grows faster than other fat tissues and it is a reliable parameter for judging total body fat content because it is directly linked to total body fat content in avian species (Tumova and Teimouri, 2010).

The present research was designed to feed the pre-determined $30 \mathrm{mg}$ of folic acid per liter of drinking water for varying durations from day-one of age of the broiler and determine the effect on performance and carcass characteristics with emphasis on abdominal fat. The objective was to confirm the high levels of abdominal fat pads recorded in the folic acid treated birds in the previous trials so as to clarify the mechanism underlying adipose tissue growth in broilers. Parameters measured were: performance (body weight, body weight gains, feed intake, folic acid intake, feed conversion ratio, feed efficiency and mortality), carcass (dressed weight, dressing percentage, breast with wings, thigh with drumstick and back), internal organs (Heart, gizzard, abdominal fat and liver).

\section{MATERIALS AND METHODS}

\section{Ethical approval}

Chickens were handled and managed in accordance with the recommendations in the Guide for the Care and use of Animals, at the Faculty of Agriculture and Forestry Obubra Campus, Cross River University of Technology, Cross River State Nigeria.

\section{Experimental site}

The research was carried out at the Teaching and Research Farm of the Department of Animal Science, Faculty of Agriculture and Forestry, Obubra Campus. Cross River University of Technology, Cross River State Nigeria. The location of the study lies along Latitude 6" 4.6032' N and Longitude 8" 19.9446' East (Date and Time Information, 2020).

\section{Experimental treatments and design}

The treatments comprised: a control and three different durations of folic acid administration from day-one of age. These includes: T1 (control) $=$ no folic acid supplementation; T2 $=30 \mathrm{mg}$ of folic acid /litre of drinking water for 7 days $(0-7$ days of age); T3=30mg of folic acid / litre of drinking water for 10 days (0-10 days of age); T4=30mg of folic acid / litre of drinking water for 14 days (0-14 days of age); Each treatment was replicated three times in a complete randomized design i.e. twelve experimental units (pens). The folic acid supplements were purchased from one of the patent medicine stores within the vicinity of the experiment. The folic acid pills were put into the drinking trough of water and allowed to dissolve before stirring with a spatula to avoid loss of particles.

\section{Management of experimental birds}

Sixty day-old broiler chicks were purchased from a commercial distributor. The birds were divided into twelve groups and each group was randomly assigned to an experimental unit. Each group was brooded separately in deep litter pens measuring $1 \mathrm{~m} \times 1.5 \mathrm{~m}$ (width $\times$ length). The birds were raised in these pens by dismantling the brooding compartments after brooding. Feed and water were given ad libitum throughout the duration of the experiment which lasted for eight weeks (56 days). Management during the brooding and rearing periods was based on standard husbandry practices for broiler production. Commercial diets were used for the experiment. Chemical compositions of the experimental diets are presented in table 1.

\section{Table 1 - Chemical composition of experimental diets for broilers.}

\begin{tabular}{lcc} 
Composition & Starter & Finisher \\
\hline Crude protein $(\mathrm{g} / \mathrm{kg})$ & 21.00 & 19.00 \\
Fat $(\mathrm{g} / \mathrm{kg})$ & 8.50 & 8.50 \\
Crude fibre (g/kg) & 5.00 & 5.00 \\
Phosphorus (g/kg) & 0.45 & 0.41 \\
Calcium (g/kg) & 1.20 & 1.20 \\
Metabolizable energy (Kcal/kg) & 2,800 & 2,900 \\
*Source: Grand Cereal and Oil Mills Limited (Jos, Plateau State Nigeria). & \\
\hline
\end{tabular}


Data collection and analysis

The birds were weighed at the beginning of the experiment and thereafter at weekly intervals. Feed offered daily were weighed and the leftover weighed the following morning. Feed intakes were obtained by subtracting the leftover from the quantity supplied the previous day. Weight gain and feed conversion ratio (FCR) were deduced from the live weight records. Mean values of folic acid consumed per bird per day were calculated by simple proportion using mean water intake per bird per day in relation to $30 \mathrm{mg}$ of folic acid per litre of drinking water. At the end of the experiment at eight weeks of age, three birds were randomly selected per treatment (one from each replicate). The birds were weighed, slaughtered and dressed for carcass analysis. The slaughtering and dressing were carried out using standard procedures for processing broilers. The internal organs were removed, separated, weighed and recorded. Data generated were analyzed using statistical package for social sciences SPSS Version 16.0 (Student's version). Significant means were separated using Duncan's Multiple Range Test of the same software.

\section{RESULTS}

The results of the performance of the experimental birds are presented in table 2, while the results of the carcasses, carcass cuts and internal organ weights are presented in table 3.

\section{Performance}

There was no difference $(P>0.05)$ in the final body weight and weight gains of the experimental birds including the control. There was also no difference $(P>0.05)$ in feed intake apart from the group on the longest duration of folic acid supplementation (14 days, T4). This group (T4) exhibited higher feed intake $(P<0.05)$ than all the other groups including the control which resulted to low feed utilization efficiency for this group i.e. higher $(P<0.05)$ feed conversion ratio. Folic acid intake increased significantly $(P<0.05)$ with increase in the duration of administration due to increase in water intake as the birds ages. Mild mortality was spread across the treatments.

\section{Table 2 - Performance of the experimental birds fed pre-determined level of folic acid for varying periods.}

\begin{tabular}{|c|c|c|c|c|c|}
\hline \multirow{2}{*}{ Parameters } & \multirow{2}{*}{$\begin{array}{l}\text { No folic acid } \\
\text { T1 (control) }\end{array}$} & \multicolumn{3}{|c|}{$30 \mathrm{mg}$ of folic acid/litre of water } & \multirow{2}{*}{ SEM } \\
\hline & & T2 (7days) & T3 (10 days) & T4 (14 days) & \\
\hline Initial body weight (g) & 40.00 & 40.00 & 40.00 & 40.00 & 0 \\
\hline Final body weight (kg) & 2.48 & 2.45 & 2.47 & 2.48 & $0.13^{\text {ns }}$ \\
\hline Feed intake (g/day) & 116.37 & 116.18 & 116.31 & 127.27 & $8.97^{\text {ns }}$ \\
\hline Body weight gain (g/day) & 44.28 & 43.75 & 44.11 & 44.28 & $0.92^{\text {ns }}$ \\
\hline FCR (g of feed/g of gain) & 2.63 & 2.66 & 2.64 & 2.87 & $0.18^{\mathrm{ns}}$ \\
\hline $\mathrm{FE}$ (g of gain/g of feed) & $0.38^{a}$ & $0.376^{a}$ & $0.38^{a}$ & $0.347^{b}$ & $0.02^{*}$ \\
\hline Folic acid intake (mg/bird/day) & 0 & 0.71 & 0.98 & 1.33 & $0.12^{\text {ns }}$ \\
\hline Mortality (\%) & 6.67 & 6.67 & 0 & 6.67 & 0 \\
\hline
\end{tabular}

\section{Carcass and carcass cuts (visual appraisal)}

The outstanding effect of the $30 \mathrm{mg}$ of folic acid supplementation on the broiler chickens was the physical manifestation on the dressed carcasses of the experimental birds (body conformation). The folic acid treated birds had expanded abdominal region that were filled with large mass of abdominal fat pads irrespective of the duration of supplementation, while the control birds had normal carcass conformation that is characteristic of normal growth. The dressed carcasses of the experimental birds are shown in the figure 1 . The result of the carcass and carcass cuts (Figure 1) followed the same trend with that of performance parameters. There was no significant difference $(P>0.05)$ between the treatments including the control in all he parameters measured, except the group on the highest duration of folic acid supplement (14 days, T4). The significant $(P<0.05)$ low feed efficiency of the T4 group (Table 2) manifested in significant $(P<0.05)$ low dressed weight and dressing percentage (Table 3$)$. The low $(P<0.05)$ dressed weight of the T4 group reflected in the thigh and drumstick.

\section{Internal organs}

Apart from the abdominal fat which was significantly $(P<0.05)$ higher in all the folic acid treated birds than the control, there was no difference $(P<0.05)$ between the control and the folic acid treated birds in all the internal organs measured. The folic acid treated birds did not differ $(P>0.05)$ in their abdominal fat irrespective of the duration of administration. 


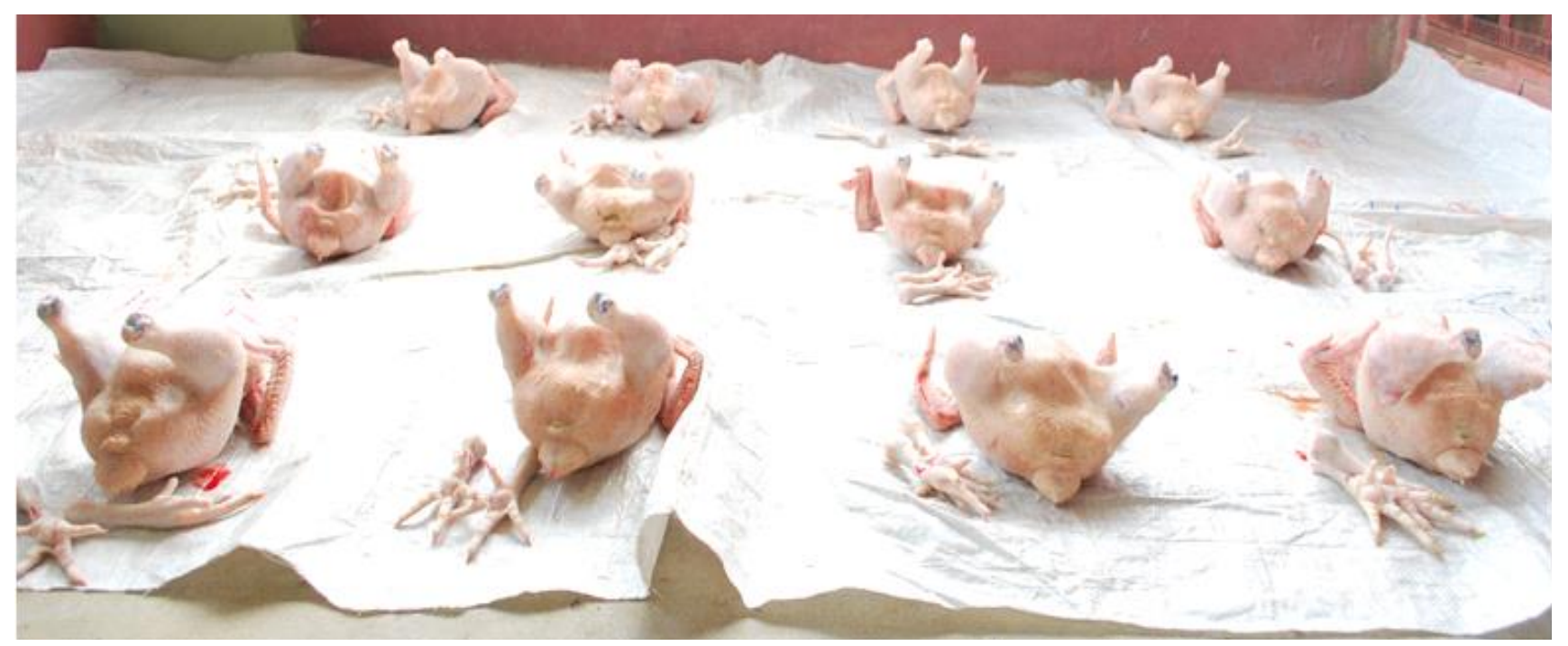

Figure 1. Dressed carcasses of the experimental birds.

\begin{tabular}{|c|c|c|c|c|c|}
\hline \multirow[b]{2}{*}{ Parameters } & \multirow{2}{*}{\begin{tabular}{|c|} 
No folic acid \\
T1 (control)
\end{tabular}} & \multicolumn{3}{|c|}{$30 \mathrm{mg}$ of folic acid/litre of water } & \multirow{2}{*}{ SEM } \\
\hline & & T2 (7days) & T3 (10 days) & T4 (14 days) & \\
\hline Pre-slaughter weight (kg) & 2.40 & 2.40 & 2.40 & 2.40 & $0.08^{\mathrm{ns}}$ \\
\hline Dressed weight (kg) & $1.744^{\mathrm{a}}$ & $1.743^{a}$ & $1.70^{\mathrm{a}}$ & $1.650^{b}$ & 0.04 \\
\hline Dressing percentage (\%) & $72.64^{a}$ & $72.62^{a}$ & $72.50^{a}$ & $68.75^{b}$ & 0.90 \\
\hline \multicolumn{6}{|c|}{ Carcass cuts (\% of pre-slaughter weight) } \\
\hline Thigh + drumstick & $24.03^{a}$ & $24.02^{a}$ & $24.00^{a}$ & $21.28^{b}$ & 0.89 \\
\hline Back & 15.28 & 15.27 & 15.20 & 14.28 & $1.27^{\mathrm{ns}}$ \\
\hline Breast + wing & 33.33 & 33.33 & 33.30 & 33.10 & $0.68^{\mathrm{ns}}$ \\
\hline \multicolumn{6}{|c|}{ Internal organs (\% of pre-slaughter weight) } \\
\hline Heart & 0.43 & 0.43 & 0.42 & 0.42 & $0.025^{n s}$ \\
\hline Liver & 1.94 & 1.94 & 1.93 & 1.93 & $0.031^{n s}$ \\
\hline Abdominal fat & $1.17^{b}$ & $3.50^{a}$ & $3.48^{a}$ & $3.50^{a}$ & 0.43 \\
\hline Gizzard & 1.65 & 1.65 & 1.66 & 1.66 & $0.03^{n s}$ \\
\hline
\end{tabular}

\section{DISCUSSION}

The folic acid supplemented birds had expanded abdominal regions that were filled with large mass of abdominal fat pads irrespective of the duration of supplementation. This result agrees with the report of Meremikwu et al. (2015) that, the folic acid treated birds in their research were dramatically different from the control birds in carcass appearance and conformation.

The enlarged abdominal regions of the folic acid treated birds that were filled with large mass of abdominal fat pads could be due to cell multiplication resulting from Folate-mediated one-carbon units transfer reactions. This is supported by the reports of Wang et al. (2007) and Guo et al. (2011) that fat growth in chicken within the first fourteen (14 days) of age is by hyperplasia (cell multiplication) after which hypertrophy (cell enlargement) of existing adipose cells becomes responsible for increases in the mass of these fat depots. This is also supported by the reports of Field (2018) and Tjong and Mohiuddin (2019) that Folate-mediated one-carbon units transfer reactions are important during periods of active cell division and support rapid proliferation of cells due to ample supply of nucleotides with many activated precursors of nucleic acid (thymidine and purine). The non-significant $(P>0.05)$ difference in carcass cuts (especially the breast) between the control and the folic acid treated birds implied that folic acid supplement given for seven to fourteen days of age of the broilers had no effect on factors that influence muscle growth in broiler. This is supported by the fact that the hyperplasic period of muscle development is said to be nearly complete at hatch (Rutz, 2015) and folic acid is reported to be effective during periods of active cell division (Armando, 2018; Tjong and Mohiuddin, 2019). The high feed intake of the group on the longest duration of folic acid could be due to the fact that folic acid is said to be an appetite stimulant when taken in high doses (Marshal, 2016). According to Marshal (2016), folic acid is a nutrient with two-edged sword i.e. it is an appetite stimulant and a hunger deregulator, in which case, if an individual is deficient in folic acid, the first thing that goes is the appetite. 


\section{CONCLUSION}

Thirty $\mathrm{mg}$ of folic acid supplement per litre of drinking water administered for 7 - 14 days from day-one of age, has confirmed the results of previous trials with graded levels of folic acid supplement by producing visual morphological effect on the dressed carcasses of the folic acid treated birds, characterized by expanded abdominal regions that were filled with large mass of abdominal fat pads. It was concluded from this research that, the excessive growth of abdominal fat depots in the folic acid treated broiler chickens were by cell multiplication (hyperplasia), due to the fact that Folatemediated one-carbon transfer reactions are important during periods of active cell division and supports high rate of cell proliferation because of many activated precursors of nucleic acid (thymidine and purine). This research has confirmed the fact that adipose tissue growth in chicken within the first fourteen days of age is by hyperplasia. It is therefore recommended that, folic acid or any other supplement that supports cell multiplication should not be used within the first 7 to 14 days of age of the broiler since this will induce adiposity. Further research should be conducted to determine the appropriate age to administer folic acid supplement to exploit the growth promoting (cell proliferation) effect to maximize muscle tissue growth in broilers.

\section{DECLARATIONS}

\section{Corresponding author}

Victoria Nnenna. Meremikwu, Ph.D., Department of Animal Science, Faculty of Agriculture and Forestry, Obubra Campus, Cross River University of Technology, Cross River State, Nigeria. Email: victoriameremikwu@yahoo.com

\section{Ethics and consent to participate}

This paper has been submitted with full responsibility of both authors following due ethical standards and there is no duplicate publication or plagiarism.

\section{Consent to publish}

Not applicable.

\section{Conflict of interest}

The authors declare that they have no competing interest.

\section{Authors' contribution}

Both authors contributed equally to the work.

\section{Avallability of data}

Data can be availed to the journal upon request.

\section{REFERENCES}

Armando F (2018). Folic acid and pregnancy. Kids Health from Nemours. Article link

Barry S (2001). Folate chemistry and metabolism. Clinical Research and Regulatory Affairs. 18(3): 137-159. Article link I https://doi.org/10.1081/CRP-100108170

Chan YM, MacFarlane AJ and Connor DL (2015). Modeling demonstrates that folic acid fortification of whole-wheat flour could reduce the prevalence of folate inadequacy in Canadian whole-wheat consumers. The Journal of Nutrition. 145(11): 2622-2629. Article link I https://doi.org/10.3945/in.115.217851

Date and Time information (2020). Geographical coordinates of Obubra in Degrees and Minutes. Link: https://dateandtime.info/citycoordinates.php?id=2328153

Fernández-Villa D, Aguilar MR and Rojo L (2019). Folic Acid Antagonists: Antimicrobial and Immunomodulating Mechanisms and Applications. International Journal of Molecular Sciences. 20(20): 4996. Article link I https://doi.org/10.3390/ijms20204996

Field MS, Kamynina E, Chon J and Stover PJ (2018). Nuclear folate metabolism. Annual Reviews of Nutrition, 38 : 219 243. Article link I https://doi.org/10.1146/annurev-nutr-071714-034441

Greenberg JA, Bell SJ, Guan Y and Yu Y (2011). Folic Acid supplement and pregnancy. More than Just Neural Tube Defect Prevention. Review in Obstetrics and Gynecology. 4(2): 52-59. PMC3218540 I PMID: 22102928

Guo L, Sun Z, Shang L, Leng Y, Wang N. and Wang HU (2011). Comparison of adipose tissue cellularity in chicken lines divergently selected for fatness. Poultry Science. 90(9): 2024-2034. Article link I https://doi.org/10.3382/ps.201000863

Marshall TM (2016). Folic acid - appetite stimulant and hunger deregulator. https://www.dr-marshall.com

Media Experts (2020). Cell Culture Media Component /Sigma - Aldrich. Article link

Meremikwu VN, Peter BM, Odey RU and Gboshe PN (2008). The Efficiency of folic acid in promoting growth in broilers. Journal of Agriculture, Forestry and Social Science. 6(1): 209-212. Article link 
Meremikwu VN. Akpet SO, Essien A. and Orok EE (2015). Effect of folic acid supplementation on Performance of broiler chickens. Journal of Agriculture, Forestry and Social Sciences. 13(1): 104-110. Article link

Poultry DVM (2020). Folic Acid Deficiency in chickens-Poultry DVM. Article link

Rutz F (2015). Conditions affecting broiler meat quality. Poultry World. Article link

SPSS (ver. 16.0). Statistical Package for Social Science. Student version for windows, Inc.

Tumova A and Teimouri A (2010). Fat deposition in broiler chickens: A review. Scientia Agriculturae Bohemica, 41 (2): 21 128. Article link

Tjong E and Mohiuddin SS (2019). Biochemistry, Tetrahydrofolate. StatPearls [Internet]. NCBI Bookshelf, Medline. Article $\underline{\text { link }}$

Wagner C (2001). Biochemical Role of folate in cellular metabolism. Clinical Research and Regulatory Affairs. 18(3):161180. Article link I https://doi.org/10.1081/CRP-100108171

Wang HB, Li H, Wang QG, Zhang XY, Wang SZ, Wang YX, Wang XP (2007). Profiling of chicken adipose tissue gene expression by genome array. BMC Genomics. 8(1): 1-4 Article link I http://doi.org/10.1186/1471-2164-8-193

Whitehead CC (2002). Nutrition and Poultry Welfare. World's Poultry Science Journal. 58(3): 349-356. Article link I https://doi.org/10.1079/WPS20020027 